\title{
DER VERSUCH AUF EINER WELLE ZU REITEN, DIE UNS ZU ÜBERROLLEN DROHTE: BESCHLEUNIGTE DIGITALISIERUNG IM BIBLIOTHEKSSCHULUNGSWESEN IM ZUGE DER COVID-19-PANDEMIE
}

\author{
von Valentin Umlauft und Anton Walder
}

Zusammenfassung: Große Änderungen wie die "digitale Wende"vollziehen sich in unzähligen kleinen Einzelschritten, selbst unter zeitlichem Druck und bei fehlender Ausrüstung. Damit das Wenden zu Online-Schulungsangeboten und-unterlagen im Bibliotheksbereich möglichst friktionsfrei erfolgen kann, braucht es weniger Theorie als lösungs-, bedarfs- und zielgruppenorientierte Praxisnähe, die durch ein „Entbündeln" der gesamten Kursinhalte zu eigenständigen Modulen erreicht werden kann. Dieser Beitrag beleuchtet die Herangehensweise des Zentrums für Informationskompetenz der Universitäts- und Landesbibliothek Tirol bei der Erstellung eines nachhaltigen digitalen Angebots vor und während der COVID-19-Pandemie, sowie den Einfluss der Dynamik der Ereignisse, die selbst zum Katalysator der Wende wurden.

Schlagwörter: Digitale Wende; Informationskompetenz; Schulungsangebot; Zielgruppenorientierung; Krisenmanagement; COVID-19

\section{TO RIDE THE WAVE THAT TRIED TO CRUSH US: ACCELERATED DIGITALIZATION IN LIBRARY EDUCATIONAL PROGRAMS DURING THE COVID-19 PANDEMIC}

Abstract: Great changes such as the transition towards digitalization happen step by step, even under time pressure and without sufficient equipment. Unbundling course contents and structures from a greater course frame to create stand-alone content, as well as focusing on the needs of target groups may ease the digital change in library course programs more than theory-based approaches. This article intends to shed light on the concepts for the creation of online library courses and educational material before and during the Corona-pandemic that were put into practice by the Zentrum für Informationskompetenz at the Universitäts- und Landesbibliothek Tirol.

Keywords: Digital transformation; Information literacy; Educational program; Target group orientation; crisis management; COVID-19

DOI: https://doi.org/10.31263/voebm.v73i3-4.5330 
Dieses Werk ist - exkl. einzelner Logos und Abbildungen - lizenziert unter einer Creative-Commons-Lizenz Namensnennung 4.0 International-Lizenz

Dass die Not erfinderisch macht, ist nur die halbe Wahrheit. Denn die Materie, die zur Erschaffung neuer Dinge vorhanden sein muss, ist und das ist die andere Hälfte der Wahrheit - eine über die Zeit gesparte Substanz. In weniger kryptischen Worten: keine Nachhaltigkeit ohne Wiederverwertung, keine Wiederverwertung ohne Originale. Noch weniger obskur: Zuerst muss etwas da sein, damit etwas anderes wachsen kann.

Schlagworte wie die "digitale Wende" suggerieren uns das geistige Bild eines Mauerfalles, eines Dammbruches, nach dem die Welt schlagartig nicht mehr so ist, wie sie war. Eine Barriere wird eingerissen, ein System generalüberholt, alte Schaltkreise ausgemustert und durch nigelnagelneue ersetzt. Was hier in der Wortverwendung allerdings verloren geht, ist dass die Wende selbst nicht die Richtung bezeichnet, in die gewendet wird, sondern nur die Bewegung an sich. Die Mittel- und Langfristigkeit einer Wende, die bereits schon durch den breiten Klang des Wortes und die Dehnung der Laute begreiflich wird, muss daher ebenfalls einkalkuliert werden: In der Bewegung des Wendens liegt eine Behäbigkeit, womöglich gilt es, sich gegen einen Widerstand durchzusetzen. Wendet ein Frachttanker auf hoher See, geschieht dies nicht in der gleichen Geschwindigkeit wie das Wenden eines Fahrrades - es ist kein rasches Umdrehen, sondern das Durchschreiten eines weiten Bogens. So erfolgt auch die digitale Wende nicht in einem abrupten Kurswechsel von heute auf morgen. Vielmehr entsteht sie - oder besteht sie - aus zahlreichen Einzelschritten, die diese weitläufige Kehrtwende beschreiben.

Der Ausgangspunkt der digitalen Wende des „Zentrums für Informationskompetenz" (ZFI), der Schulungs- und Fortbildungsabteilung an der Hauptbibliothek der Universitäts- und Landesbibliothek Tirol (ULBT), liegt für die beiden Autoren im Feber 2019.

\section{Ausgangssituation}

Bereits ab offiziellem Tätigkeitsbeginn des ZFI bestand der Wunsch, den drei großen Zielgruppen der ULBT (Studierende/Forschende/Lehrende; 
Schüler*innen, sowie die interessierte Öffentlichkeit) das bereits bestehende Schulungsangebot in digitaler Form zu präsentieren. Dieses vorerst viel zu vage gefasste Ziel ließ sich nicht so zügig umsetzen, da einige entscheidende Voraussetzungen (noch) nicht gegeben waren:

- Die Konzeption des Angebotes war ausschließlich auf das Abhalten von Vorträgen und Workshops ausgelegt.

- Die technische Ausrüstung und das Knowhow fehlten.

- Kursbegleitende Unterlagen waren nicht vorhanden.

- Organisatorische Barrieren konnten nicht überwunden werden (z. B. keine Berechtigung zur Bearbeitung der Homepage, um ggf. Unterlagen digital bereitzustellen).

In dieser Situation kam es gelegen, dass die bestehenden Angebote und Inhalte des Schulungsprogramms während der Startphase entbündelt ${ }^{1}$ und auf ihre Nützlich- und Anwendbarkeit für die jeweiligen Zielgruppen abgeklopft und segmentiert wurden. Diese Grundbausteine oder Module (z. B. Bedienung von PRIMO, DBIS, spezifische Fachdatenbanken, EZB, Nominalkatalog, VPN, Literaturverwaltung, etc.) wurden im nächsten Schritt verschriftlicht, woraus sich unabhängige, autarke Unterlagen und Anleitungen entwickeln ließen, die selbst wiederum weitere Anstöße für neu-erschließbare Themenbereiche gaben. So wurden im Verlauf des Jahres 2019 zahlreiche Materialien entwickelt, die aber noch mangels praktikabler digitaler Plattform den größten Teil ihres Daseins in Schubladen fristeten.

Die auf ersten Blick „nur für die Schublade“ produzierten Unterlagen bildeten später allerdings ebenjene Elemente, aus denen sich im März 2020 die Online-Tutorials formen ließen. Weiters regte die Erstellung der Materialien nützliche (Um-)Denkprozesse an: Erstens erfolgte durch die konzentrierte Beschäftigung mit den Inhalten und durch die Verschriftlichung der Erklärungsansätze für Benutzer*innenfragen eine Steigerung der didaktischen Qualität der Schulungen bei zeitgleicher Straffung der insgesamten Kursdauer, zweitens führten die Tätigkeiten zur Einsicht, dass es nicht „ein" Schulungsangebot im Singular gibt.

Die Entbündelung der Schulungsbausteine führte also direkt zur Erstellung eines dualen Angebotskonzepts, in welchem alle vorhandenen Materialien in zwei Strukturen verwertet werden konnten: 


\begin{tabular}{|c|c|c|}
\hline & $\begin{array}{l}\text { Struktur 1: } \\
\text { Kurse }\end{array}$ & $\begin{array}{l}\text { Struktur 2: } \\
\text { Unterlagen }\end{array}$ \\
\hline Formate & $\begin{array}{l}\text { Vorträge, Workshops, } \\
\text { Beratungseinheiten }\end{array}$ & $\begin{array}{l}\text { Anleitungen, Tutorials, } \\
\text { Leitfäden, FAQs }\end{array}$ \\
\hline Verfügbarkeit & Auf Anfrage nach Termin & Ständig verfügbar \\
\hline Zielgruppen & $\begin{array}{l}\text { Lehrveranstaltungen, } \\
\text { Klassen, Gruppen, } \\
\text { Einzelpersonen }\end{array}$ & $\begin{array}{l}\text { Vorrangig: } \\
\text { Studierende als } \\
\text { Einzelpersonen }\end{array}$ \\
\hline $\begin{array}{l}\text { Inhaltliche } \\
\text { Gestaltung }\end{array}$ & $\begin{array}{l}\text { Auf die jeweilige Zielgruppe } \\
\text { abgestimmt, d. h. } \\
\text { modulares System, nach } \\
\text { Studien- bzw. Fachrichtung } \\
\text { ausdifferenziert. }\end{array}$ & $\begin{array}{l}\text { Alle Module als getrennte } \\
\text { Unterlagen; weitere } \\
\text { Ausdifferenzierungen nach } \\
\text { Studien- bzw. } \\
\text { Fachrichtungen. } \\
\text { Ergänzen und erweitern die } \\
\text { Struktur } 1 \text { (Kurse). }\end{array}$ \\
\hline $\begin{array}{l}\text { Interaktions- } \\
\text { möglichkeiten } \\
\text { für Nutzer*innen }\end{array}$ & $\begin{array}{l}\text { Während des Kurses } \\
\text { eingeplanter Raum für } \\
\text { Fragen; nach Kursen via } \\
\text { E-Mail usw. }\end{array}$ & E-Mail usw. \\
\hline $\begin{array}{l}\text { Plattform } \\
\text { (vor 2020) }\end{array}$ & $\begin{array}{l}\text { In den Schulungsräumen der } \\
\text { ULBT, in Hörsälen, etc. }\end{array}$ & $\begin{array}{l}\text { Nach Kursen weitergereichte } \\
\text { Unterlagen, da noch keine } \\
\text { digitale Plattform } \\
\text { vorhanden war. }\end{array}$ \\
\hline
\end{tabular}

Die Materialien, die im Laufe des Jahres 2019 erarbeitet wurden, wurden entweder als kursunabhängige, kursbegleitende, oder als kurserweiternde Ressourcen genutzt. Letztere stellten eine Rückkoppelung zum Kursprogramm dar, da sie als eigenständige Kursbausteine/Module in unsere Schulungen zurückflossen, während die ersten beiden Formen im Rahmen von Kursen verteilt bzw. versendet wurden. Die Frage nach einer geeigneten digitalen Plattform für die Ressourcen wurde vorläufig noch nicht beantwortet. Zwar ist die Digitalisierung des bibliothekarischen Kursangebots im Entwicklungsplan² der Leopold-Franzens-Universität (LFU) vorgesehen, jedoch 
befanden wir uns erst in der Ausarbeitungsphase der vorgesehen Materialien; die Auswahl der Plattform war zu diesem Zeitpunkt nachrangig.

\section{Lockdown ante portas}

Im März 2020 wurde die Beantwortung dieser Frage akut. Unmittelbar in der Woche vor dem ersten großen Lockdown verdichteten sich die Anzeichen dafür, dass die Bibliothek geschlossen werde. Uns war vollkommen klar, was das für unsere Nutzer*innen bedeuten würde: Sie wären vollkommen vom Zugang zu Printwerken abgeschnitten. Es blieb ihnen somit nur mehr die Alternative, das Angebot an E-Medien der ULBT zu nützen. Deshalb gingen unsere ersten Überlegungen dahin, Tutorials und Schulungsunterlagen für ein vorläufiges Notfallprogramm zu erstellen.

Zuerst mussten alle Kanäle identifiziert werden, über die unsere Nutzer*innen Zugang zu digitalen Medien erhalten können: BibSearch (so wird Primo an der ULBT genannt) und Volltextdatenbanken, die in Primo nicht indexiert werden, EZB, u. dgl.

Damit war eine erste Stoßrichtung des Notfallprogramms festgelegt, Tutorials für die Literatursuche in BibSearch und Anleitungen zum Download der dort erzielten Treffer zu erstellen, sowie Unterlagen für den Umgang mit Volltextdatenbanken, die an der ULBT lizensiert oder verfügbar sind, auszuarbeiten. Dazu musste auch eine Anleitung geschaffen werden, wie die Fernnutzung via VPN-Zugang erfolgt, damit die Ressourcen genutzt werden konnten; die Universität und die Bibliothek werden ja geschlossen, so die Grundannahme.

Diese Überlegung führte zu einem weiteren wichtigen Punkt. An der ULBT haben nicht alle Zielgruppen im gleichen Maße Zugang zu den E-Medien. Ein Fernzugriff ist nur für Angehörige der LFU und der Medizinischen Universität Innsbruck (MUI) möglich. Für unsere allgemeinen Benutzer*innen (z. B.: Angehörige der Tiroler Fachhochschulen, Schüler*innen, interessierte Öffentlichkeit) musste eine Alternativlösung erarbeitet werden, die sich hauptsächlich aufdas Thema Open Access und freie Ressourcen fokussierte. Damit sämtliche Zielgruppen derULBT - so unsere Überlegung - für die Lockdown-Phase gerüstet wären, setzten wir einen programmatischen Schwerpunkt auf die bestmöglichen Such- und Nutzungsweisen von BibSearch und Datenbanken, sowie den darin vorhandenen Eingrenzungsmöglichkeiten auf freie Materialien und OA-Angebote. Darüber hinaus verfolgten wir den Ansatz, mithilfe des Notfallprogramms auf weitere Zugänge zu freien Ressourcen aufmerksam zu machen, z. B. Eingrenzung auf freie Volltexte 
im Österreichischen Verbundkatalog, Verwendung der Metasuchmaschine BASE, Anleitung zu Google Scholar, etc.

Das übergeordnete Ziel war, Unterlagen zu schaffen, die einen möglichst großen Nutzen für möglichst viele Zielgruppen gleichzeitig beinhalteten und in Folge innerhalb einer Zielgruppe möglichst viele Fachrichtungen abdeckten. In Hinblick auf diese Zielvorstellung begannen wir die noch in Schubladen schlummernden Schulungsunterlagen dahingehend umzuarbeiten und zu erweitern, dass sie alleinstehend als Lehr- und Lernunterlagen verwendet werden konnten; erklärende Schulungen waren zu diesem Zeitpunkt nicht mehr möglich und Online-Schulungen noch nicht konzipiert. So traf uns die Entwicklung der Ereignisse nicht völlig unvorbereitet, wenngleich noch vieles zu erledigen war. Beispielsweise testeten wir am Donnerstag, 12. März, die Technik des Screencastings für VideoTutorials und erstellten damit gleich eine erste Anleitung zur Nutzung des VPN-Zugangs. In der noch verbleibenden Zeit konnten wir Anleitungen zu sechs verschiedenen Fachdatenbanken und die noch in der Vorwoche fertiggestellten BibSearch FAQs für den digitalen Gebrauch adaptieren.

Der größte Knackpunkt war aber die immer noch nicht beantwortete Frage nach der geeigneten Plattform, die nun nach einer unmittelbaren Lösung drängte. Vor allem sollte es eine Lösung sein, die von allen Zielgruppen gleichermaßen ohne Zugangsbeschränkung genutzt werden konnte, waren doch die Unterlagen zu einem guten Teil auch für Nichtangehörige der Universitäten geplant. Abgesehen davon sollte es sich um ein Datenportal handeln, das wenigstens einer Zielgruppe bereits bekannt war und ohne großen Aufwand in kurzer Zeit bespielt und beworben werden konnte.

Um die Homepage der ULBT umzuarbeiten, fehlten Zeit und Zugang an dieser Stelle eine Empfehlung: Mitarbeiter*innen, die an Kursinhalten arbeiten, benötigen unbedingt die Möglichkeit, an der Homepagegestaltung aktiv mitwirken zu können oder einen adäquaten Zugang zu einer alternativen Plattform (z. B. in Form einer eigenen Unter- bzw. Projektseite).

Diese Alternative fanden wir in der Lernplattform Open OLAT, die an der LFU Innsbruck in der digitalen Lehre verwendet wird. Open OLAT sollte sich als geradezu ideal erweisen, da die Plattform unter den Studierenden und Lehrenden bestens bekannt war und zudem über eine Gastzugangsmöglichkeit verfügt, welche alle User*innen barrierefrei nutzen können. Damit fehlte letztlich nur noch der Zugang, um Kurse in OLAT zu erstellen und verwalten. Kurzerhand sprach unsere Abteilungsleiterin direkt beim Zentralen Informatikdienst (ZID) vor und wir erhielten den dringend benötigten Zugang, um unsere ersten Unterlagen hochzuladen. Zeitlich gesehen wird uns dieser Moment wohl als höchst dramatisch in Erinnerung bleiben, wenngleich wir im 
Nachhinein auch behaupten könnten, wir hätten das alles so geplant: Am Freitag, den 13. März 2020, um 11:49 Uhr erhielten wir die Open OLAT-Berechtigungen, um 12:00 Uhr schloss der ZID, am Samstag offenbarte sich der Bedarf nach Nudeln und Klopapier, am Sonntag wurde über Tirol die Ausgangssperre $^{3}$ verhängt - der erste Lockdown hatte begonnen.

\section{Von Lockdown zu Lockdown}

Über die kommenden Tage und Wochen wurde der OLAT-Kurs vom Homeoffice aus zügig erweitert. Während der schrittweisen Ergänzung der PDF- und Video-Tutorials zu den Themenbereichen „Zugang zu E-Medien“, „Datenbanken“, „Literaturrecherche“ und „-verwaltung“, sowie der Übersetzung zahlreicher Materialien ins Englische, galt weiterhin der Grundsatz, allen Zielgruppenbedürfnissen gleichermaßen nachzukommen.

Daneben begannen Ende März auch die ersten direkten Online-Schulungen im Rahmen zweier Lehrveranstaltungen und mehrerer Beratungsgespräche. Dabei wurden die von Helmut Dollfuß beschriebenen Empfehlungen ${ }^{4}$ intuitiv vorweggenommen und im Verlauf der nächsten regulär-geplanten Kurseinheiten als interne Richtlinien für das Schulungswesen adaptiert. Als Schulungsplattform wurde BigBlueButton gewählt, da diese von der Universität Innsbruck verwendet wird, relativ leicht zu bedienen ist und in den OLAT-Kurs integriert werden kann. Abgesehen davon wurden mit anderen Plattformen (Zoom, Adobe Connect, Skype for Business) negative Erfahrungen gemacht, insbesondere im Bereich der nahtlosen Audio- und Video-Übertragung sowie der Benutzungsfreundlichkeit, was allerdings letztlich aufgrund der großen Ähnlichkeit der verschiedenen Konferenzangebote eher als Geschmacksfrage gewertet werden kann.

Es wurde bewusst davon abgesehen, vollständige Schulungen aufzuzeichnen bzw. in kompletter Einheit als Video-Tutorials über den OLAT-Kurs anzubieten. Die Begründung hierfür ist, dass bei einer derartigen Kursaufzeichnung aufgrund der darin benötigten Allgemeingültigkeit keine zielgruppenspezifischen Inhalte behandelt werden können, der laufende Bearbeitungs- und Aktualisierungsaufwand zu zeit- und personalintensiv ist, den Zuseher*innen keine direkte Interaktions- und Nachfragemöglichkeiten geboten werden können und einzelne Teilinhalte ohnehin schon als entbündelte Kurz-Tutorials angeboten werden. Daher empfehlen wir den Verzicht auf die Wiederverwendung von Schulungsaufzeichnungen als eigenständige Unterlagen; sie können die Live-Schulungen (in Präsenz oder online) nicht ersetzen. 
Bei Rückkehr aus dem Homeoffice im Mai erfolgte die Revision der Kursunterlagen. Dabei wurde festgestellt, dass die Struktur des OLAT-Kurses zwar sinnvoll gewachsen war, jedoch orteten wir strukturelle Verbesserungsmöglichkeiten, insbesondere den logischen Zusammenhang der Module betreffend. Daher entwarfen wir einen neuen Aufbau, der sowohl Platz für neu-zu-erschließende Themenbereiche (z. B. Evaluierung wissenschaftlicher Quellen, Bibliometrie, Umgang mit Fake News, etc.) eröffnen, wie auch die Interaktionsmöglichkeiten der Nutzer*innen mit dem ZFI ermöglichen soll. Gleichzeitig soll in der Struktur Raum geschaffen werden, der neben der Ablage allgemeingültiger Materialien eine weitere inhaltliche Ausdifferenzierung und eine Auftrennung nach Fachbereichen benutzer* innenfreundlich zulässt.

Mit dem Plan zur Umstrukturierung fiel auch die Entscheidung, den OLAT-Kurs von einem Notprogramm in ein Regulärprogramm überzuleiten. Dieser Transformationsprozess und die damit einhergehende Strukturänderung wird wohl letztendlich erst nach dem jetzt noch nicht absehbaren Ende der COVID-19-Pandemie erfolgen können. Nun, da dieser Artikel entsteht, befinden wir uns im 2. Lockdown und der Bedarf eines Notfallprogramms ist an den wieder stark wachsenden Zugriffszahlen der Nutzer*innen unseres OLAT-Kurses klar ersichtlich. Daher wurde noch kein zeitlicher Rahmen für die Änderungen des Kursaufbaus gesetzt.

Auch die Gestaltung der Online-Schulungen hat sich seit März erheblich gewandelt: Ab Juni wurden digitale Fixveranstaltungen von Basis- und Aufbaukursen eingeführt, die nun im zweiwöchentlichen Rhythmus stattfinden und zahlreiche Besucher*innen aus allen Zielgruppen anlocken. Ergänzt wird dieses Angebot durch regelmäßige Schulungen in Literaturverwaltungsprogrammen. Die bereits vor COVID-19 bestehende Möglichkeit für Lehrveranstaltungsleiter*innen, Lehrer*innen und Gruppenleiter*innen, zielgruppenspezifische Kurse zu buchen, wurde beibehalten; momentan finden sämtliche Schulungen digital über BigBlueButton statt. Das Beratungswesen (besonders bei komplexen Rechercheanfragen und Anfragen zur Literaturverwaltung) wird ebenfalls digital abgewickelt, wobei hier der Plan besteht, zusätzlich fixe Sprechstunden einzuführen, die über ein „virtuelles Klassenzimmer" (= digitaler Konferenzraum von BigBlueButton) in unserem OLAT-Kurs eingebunden sind.

\section{Nach COVID-19}

Mit der Verwendung digitaler Lösungen hat sich auch unser bereits vor COVID-19 bestehendes, duales Angebotskonzept verändert: Beide Struk- 
turen (Kurse und Unterlagen), obwohl voneinander unabhängig, verweisen nun gegenseitig aufeinander und ergänzen sich inhaltlich wie didaktisch.

Das duale Angebot soll auch nach COVID behalten und letztendlich in ein Hybridmodell bzw. Blended Learning umgestaltet werden, in welchem alle verfügbaren Formate der physischen und digitalen Lehre und Zielgruppenunterstützung berücksichtigt werden können. Wir verstehen dies als Keimzelle für ein zukunftstaugliches Schulungsprogramm, das auf drei Säulen stehen soll: Live-Schulungen, Online-Schulungen und für beide begleitende, digital verfügbare Unterlagen. Obwohl wir bisher sehr gute Erfahrungen mit Webinaren und digitaler Lehre gemacht haben, soll in der Zeit nach COVID-19 das Hauptaugenmerk wieder auf der Präsenzlehre liegen. Das Online-Angebot wird dann als Backup dienen, um Zielgruppen zu erreichen, die weiter entfernt sind (z. B. Mechatronikstudiengang der LFU und UMIT in Lienz, Tourismuslehrgang der LFU und UMIT in Landeck, Schulklassen in Reutte, etc.), bzw. bei knappen Raumressourcen mehrere Schulungsanfragen gleichzeitig bedienen zu können. Auch auf ausdrücklichen Wunsch sollen Webinare weiterhin vereinbart werden können. Parallel dazu sollen die Schulungsunterlagen im OLAT-Kurs laufend angepasst, aktualisiert und online verfügbar gehalten werden, um alle Schulungen, seien sie live oder digital, zu begleiten.

Mit der wiedererlangten Möglichkeit zur Abwicklung der Schulungen in beiderlei Form und mit Fertigstellung unserer neuen OLAT-Kurs-Unterlagenstruktur sehen wir den wichtigsten Teil des Transformationsprozess hin zum digitalen Angebot als abgeschlossen an - zwischenzeitliche technische Neuerungen, Erschließung neuer Themenbereiche und erweiterte Möglichkeiten zur Zielgruppenbetreuung nicht inbegriffen.

Einige Erkenntnisse wurden schon jetzt aus der unmittelbaren Erfahrung der Umstellung auf unterschiedlich gestaltete Online-Angebote gewonnen:

- Um einen Transformationsprozess à la "digitale Wende“ der Bibliotheksschulungsangebote zu initiieren, braucht es einen bereits vorhandenen Vorrat an Kursmaterial, der als Treibstoff den Motor der Entwicklung am Laufen hält und zeitgleich als Nährboden für weitere Innovationen zur Verfügung steht.

- Aus den genannten Materialien können Kurse und Unterlagen abgeleitet, aus Kursen neue Unterlagen entwickelt werden und aus Unterlagen neue Kurse hervorgehen. Ein Angebot kann somit in relativ kurzer Zeit wachsen. Allerdings sollte es nicht wuchern: Eine vollkommen flächendeckende „Verkursung“ aller Unterlagen oder eine „Verunterlagung“ aller Kurse ist nicht sinnvoll, effektiv, oder zielgruppenorientiert. 
- Die Umstellung auf und das Aufrechterhalten digitale(r) Angebote sind nicht weniger zeit-, personalressourcenbindend und arbeitsintensiv als das Durchführen eines physischen Programms, sondern mehr. Schließlich müssen sämtliche Kursunterlagen laufend revidiert und angepasst werden, z. B. änderten die Datenbanken PubMed und JSTOR bzw. die Webapplikation von EndNote in der Zeit zwischen März und November 2020 jeweils mindestens einmal ihre Oberfläche oder führten neue Funktionen und Features ein. Die Konzeption, Gestaltung und Betreuung eines Online-Angebots setzt keine Personal- und Zeitkapazitäten frei, sondern bündelt diese noch stärker als die herkömmliche Lehre. Wir weisen die Meinung, dass die Digitalisierung zu einer Arbeitsreduktion führt, als Mythos zurück.

- Dahingehend lässt sich jedoch feststellen, dass sich mithilfe der Ressourcenbündelung im digitalen Bereich eine erheblich größere Anzahl an Schulungsteilnehmer*innen und Bibliotheksbenutzer*innen effizient betreuen und mit Informationen versorgen lässt. Für die Zielgruppen tritt außerdem eine zeitliche und örtliche Entkoppelung ein, was die Notwendigkeit der physischen Anwesenheit und zeitlichen Gebundenheit zu einem großen Teil aufhebt. Das digitale Angebot hat sich besonders in der Krisenzeit als praktisch erwiesen - sowohl für die Benutzer*innen (z. B.: bessere Zugriffsmöglichkeiten auf Unterlagen trotz geschlossener Bibliothek, flexiblere Kursgestaltung, etc.), als auch für uns als Mitarbeiter*innen selbst (z. B.: Betreuung aus dem Homeoffice, Abhalten mehrerer Kurse zur selben Zeit, etc.).

- Obwohl wir die Implementierung, Fortführung und Weiterentwicklung eines digitalen Angebots auch in der Zeit nach COVID für unerlässlich halten, streben wir dennoch die „Wiederbelebung“ des physischen Schulungswesens als Herzstück eines Hybridmodells an. Unsere Begründung hierfür ist, dass ein Kurs oder eine Beratung aus mehr besteht denn einem reinen Wissenstransfer. Obwohl wir in Anlehnung an Helmut Dollfuß' Ausführungen darauf achten, den digitalen Schulungen via Webcam-Funktion ein "Gesicht zu geben“" kann ein kleines Fenster auf einem Bildschirm niemals vollständig die Gestik und Mimik, Stimmgestaltung und Ausstrahlung von Vortragenden oder Zuhörenden - kurz: die menschliche Komponente - ersetzen.

Abschließend muss gesagt werden, dass die COVID-Pandemie die Digitalisierung der bibliothekarischen Kurse der ULBT nicht etwa nur beschleunigt hat, sie war genaugenommen der entscheidende Impuls, die gesamten 
Kursinhalte und -formate neu aufzustellen. Auf Krisensituation und Lockdown folgten nicht etwa Schockstarre und Resignation, die Wucht der Ereignisse konnte in ein neu gestaltetes Schulungsprogramm kanalisiert werden, die Welle wurde geritten, die alles zu zermalmen drohte.

Valentin Umlauft Universität Innsbruck, Universitäts- und Landesbibliothek Tirol E-Mail: valentin.umlauft@uibk.ac.at

Mag. Anton Walder Universität Innsbruck, Universitäts- und Landesbibliothek Tirol E-Mail: anton.walder@uibk.ac.at

\section{Literatur}

Adams Becker, S. et al. (2017): NMC Horizon Report: 2017 Library Edition, Austin. https://library.educause.edu/resources/2017/2/2017-horizonreport

Arora, Steffen (2020): Tirol Steht Still. Der Standard, 16.03.2020, S. 5.

Dollfuß, Helmut (2020): Virtuelles Treffen der VÖB-Kommission Informationskompetenz. Mitteilungen der Vereinigung Österreichischer Bibliothekarinnen \& Bibliothekare 73, Heft 2, S. 203-206. https://doi. org/10.31263/voebm.v73i2.3982

Universität Innsbruck (2017): Entwicklungsplan 2019-2024. https:// www.uibk.ac.at/universitaet/leitung/dokumente/entwicklungsplan-2019-2024.pdf

1 Bei der Entbündelung unseres Programms beriefen wir uns auf S. Adams Becker, Cummins M., A. Davis u. a., NMC Horizon Report: 2017 Library Edition, Austin 2017, S. 34, wobei wir eine engere Interpretation des im Bericht angesprochenen "to unbundle library services" wählten.

2 Vgl. Universität Innsbruck, Entwicklungsplan 2019-2024, Innsbruck 2017, S. 57.

3 Vgl. Steffen Arora, Tirol steht still, in: Der Standard, 16.03.2020, S. 5.

4 Vgl. Helmut Dollfuß: Virtuelles Treffen der VÖB-Kommission Informationskompetenz. Mitteilungen der Vereinigung Österreichischer Bibliothekarinnen \& Bibliothekare 73 (2020), Heft 2, S. 203-206, hier S. $205 f$.

5 Ebenda, S. 205. 